\title{
Data analysis and presentation in flow cytometry
}

\author{
Ladawan Khowawisetsut $^{\mathrm{a}}$, Kasama Sukapirom ${ }^{\mathrm{b}}$, Kovit Pattanapanyasat ${ }^{\mathrm{b}, *}$ \\ a Department of Parasitology, Faculty of Medicine Siriraj Hospital, Mahidol University, \\ Bangkok 10170 Thailand \\ b Centre of Excellence for Flow Cytometry, Department of Research and Development, \\ Faculty of Medicine Siriraj Hospital, Mahidol University, Bangkok 10170 Thailand
}

*Corresponding author, e-mail: kovit.pat@mahidol.ac.th

\begin{abstract}
The advancement of flow cytometry technology together with a series of novel developments in hardware and software have facilitated both phenotypic and functional characterizations of different cell types. In addition, the availability of many monoclonal antibodies and an expanding range of dye-chemistry have made multi-parameter flow cytometry possible for simultaneous measurements of large numbers of cells with better information of complex cellular networks such as the immune system. Although it has the advantage of being a fast, objective and quantitative, but running polychromatic flow cytometry is a complex process with many challenges particularly in the data analysis. The purpose of this communication is to describe several types of presentation and analysis of both univariate and multivariate datasets.
\end{abstract}

KEYWORDS: multivariate, statistics, univariate

\section{INTRODUCTION}

Flow cytometry is a technology that allows rapid and simultaneous measurements (-metry) of multiple physical and chemical characteristics of particles or single cells (cyto-) in a mixed cell populations flowing in a fluid stream (flow) that delivers the cells one by one by means of hydrodynamic focusing of cells that pass a point in a flow system where they are interrogated by optical and electronic sensors $^{1-4}$. Flow cytometry has become a powerful tool for biological research and clinical diagnostics, and its applications have been essential to innumerable advances in cell biology and immunology, as well as for understanding diseases such as immunodeficiency and cancer ${ }^{5,6}$. With an advent of monoclonal antibodies and an increasing number of fluorescent dyes, together with continuing improvements in the computerized hardware and associated software, the development of reliable techniques for performing polychromatic flow cytometry analysis has been possible ${ }^{7-10}$. At present, there are over 50 vendors in the flow cytometry business, selling flow cytometers ranging from high-end of up-to 21parameter systems to just one or two-colour pointof-care systems. It is therefore not surprising that the number of publications using flow cytometry has increased dramatically during the past two decades.

The immune system stockpiles a huge arsenal of immune cells which display a huge diversity with hundreds of discrete subsets even within the same lineage, such as T, B, and NK lymphocytes. Identification of such heterogeneity can be only achieved through polychromatic flow cytometry that allows the analysis of multiple cell membrane and intracellular molecules at the level of single cell lineage. Flow cytometric analyses thus constitute an important step towards an understanding of the complex immune system. Theoretically, as many as 161024 possible subphenotypes can be identified in a single sample stained with 4-10 fluorochromeconjugated monoclonal antibody reagents; and the number of variables can also be perplexing if the different experimental conditions or in clinical studies involving patients in different treatment regimens are applied ${ }^{10,11}$. Such large datasets can be analysed by the conventional approach of sequential gating and the representation of a particular cell subset expressing marker(s) of interest are then determined. For almost two decades, our laboratory has been using both simple $3 / 4$ to advanced 8/14colour flow cytometer to identify the phenotype and functional characteristics of peripheral blood immune cells from patients with infection ${ }^{12-15}$. We also provide basic and advanced flow cytometry courses for graduate students. The courses are given by our staff who share their in-depth experience of this evolving technology. These lecture and hand-on based courses designed to build up knowledge through step-by-step experiments to ensure that the students understand the basic elements of flow cytometry and have invaluable experience 
in designing experiments, executing, analysing and presenting the data. In this report, we review several types of presentation and analysis of the flow cytometric data by discussing how the data are processed, displayed and interpreted.

\section{DATA DISPLAYS}

Once the cells of interest are passed sequentially through the light source, the scattered light and fluorescence of different wavelengths elicited from the cells by the laser are recorded and converted to electrical pulses by collection optics or photodetectors. In general, flow cytometer has three types of photodetectors: (1) a forward scatter (FSC) detector is a relatively sensitive photodiode set behind an obscuration bar and detects light scatter in a forward direction of the laser beam. The intensity of this scatter signal depends on the cross-sectional area of the cell, i.e., the size. (2) The $90^{\circ}$ light scatter or side scatter (SSC) detector collects refracted and reflected light signals, which are proportional to cell complexity or granularity inside the cell. (3) Fluorescent light is received by a lens and divided between several photomultiplier tube (PMT) detectors either by a dichroic beam splitter or a semi-silver mirror. The electrical pulses are then processed by a series of linear and logarithmic amplifiers, and finally converted to channel numbers by the analogue-to-digital converter (ADC). Data accumulated using the flow cytometer can be analysed using the manufacturer own software, e.g., CELLQuest Pro, FACSDiva, CytoExpert, KaluZA, etc. The data outputs can also be stored in the form of computer files using flow cytometry standard (.FCS) format file extension developed by the International Society for Advancement of Cytometry (ISAC). The structure of .FCS is divided into Header, Text, and Data. The Header segment is used to identify the file as an FCS file and specify the version of .FCS used. Several keywords and numerical values in the Text segment describe the sample and the experimental conditions. Data segment is applied for numerical values in a list mode file format specified in the Text segment. It should be noted that the current version of .FCS file format is FCS 3.1 (ISAC Data Standards Task Force) ${ }^{16}$. Once data corresponding to one sample are collected, there is no need to stay connected to the flow cytometer and analysis is most often performed on a separate computer. This is necessary especially in core facilities where usage of the flow cytometers is in high demand. The data storage file includes a description of the sample acquired, the instrument setting on which the data was created, the data set, and the results of data analysis. As there are numerous flow cytometry systems in the market, a well-defined and uniform file format is therefore important as it allows data acquired by computer from any flow cytometer to be correctly exported and analysed by software on other computers running a variety of operating systems. At present there are many free flowing data analysis software, e.g., FLOWING SofTWARE, WINMDI, web-based CYTOBANK, CyTOSPEC, etc. Catalogue for free flow cytometry software can be obtained from Purdue University Cytometry Laboratories (www.cyto.purdue.edu). There are also many commercial software products, but the popular software package for analysing flow cytometry data is FLOWJo software (Tree Star, Ashland, OR; www.flowjo.com), a Windows version with web portal MyCyte.org.

The data generated by any instruments can be plotted in a single dimension, to produce univariate histograms, or in bivariate histograms such as dot plots, density plot, contour plots, chromatic plots, isometric plots, or even in three dimensions (Fig. 1). Univariate histogram is the simplest of all ways to display data with a list of the events corresponding to the graphical display specified in the acquisition protocol. It can be in the form of one-histogram or two-histogram files. A single parameter can be displayed as a single histogram, where the horizontal $(x)$ axis represents the parameter's signal value in channel numbers and the vertical $(y)$ axis or ordinate represents the number of events per channel number. These channels correspond to the original voltage generated by a scattered fluorescence detected by the PMTs. Thus the higher channel number is related to a higher pulse height or brighter specific fluorescent events. A graph with two histograms can also be shown simultaneously in a plot in which one histogram is displayed on the $x$-axis and the other histogram is displayed on the $y$-axis. In two-parameter or bivariate plots, one parameter is displayed on the $x$-axis and the second parameter is plotted on the $y$-axis, and the cell counts or events are displayed as a density (dot) plot or contour map. The parameters could be FSC, SSC, or fluorescence. Displaying three-dimensional plot by adding the third parameter being the number of cells is great for presentation and helps clarifying different cell clusters, but it is not useful for data analysis. The FLowJo's 3D Tool displays three parameters of the .FCS data simultaneously. Viewers can adjust the viewing angle by clicking and moving with the mouse, or use sliders in the interface to 

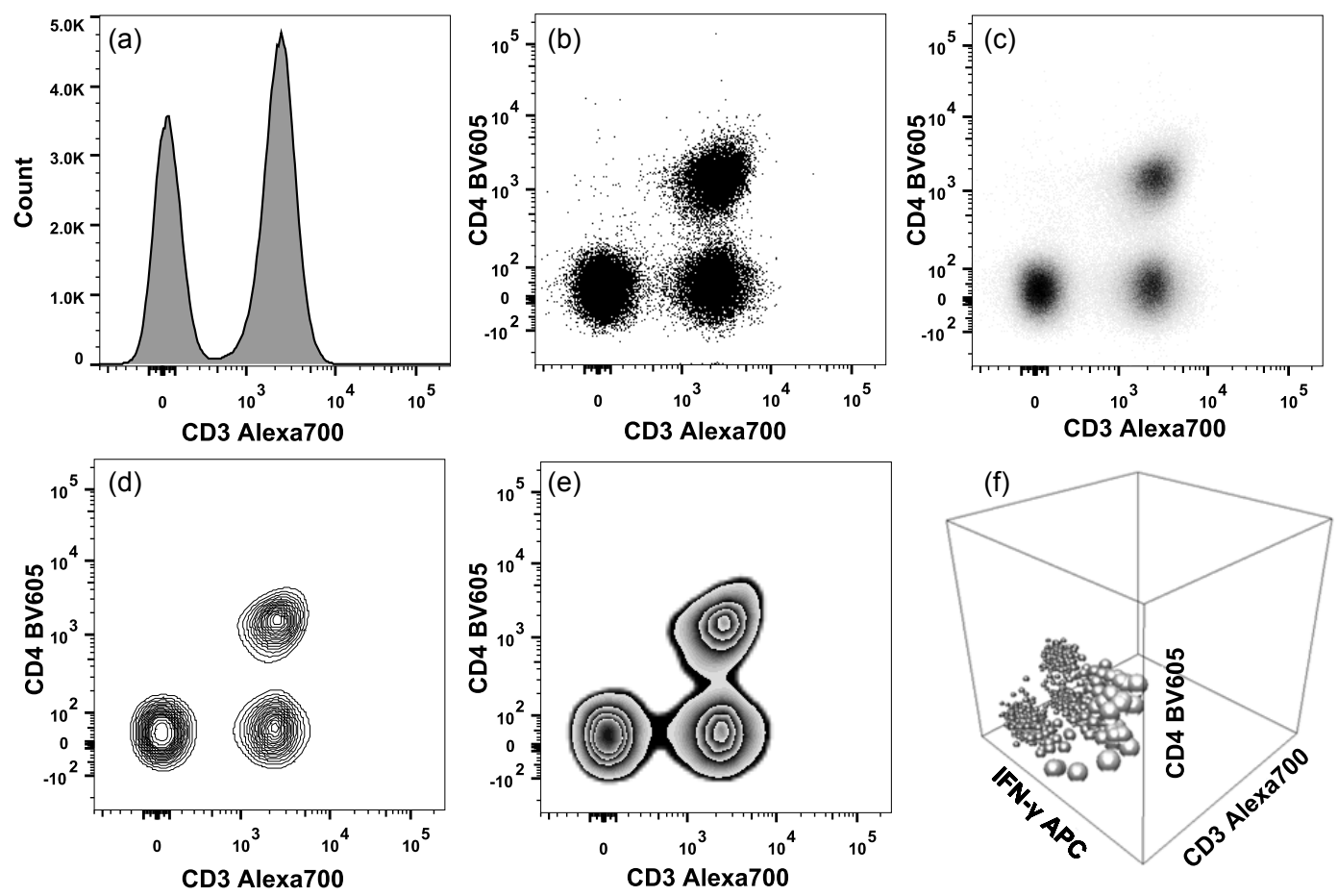

Fig. 1 Flow cytometric data can be displayed as (a) histogram, (b) dot-plot, (c) density plot, (d) contour plot, (e) zebra plot as well as (f) 3-dimension plot.

rotate the display of each parameter on $x, y$, or $z$ axis. Besides using a graph to present several parameters of single cell population, multigraph is another useful option to display all parameters from cell population. Multigraph overlays or $\mathrm{N} \times \mathrm{N}$ plots allow the visual comparison of the same parameters of multiple cell subpopulations of the same sample or the same parameters of one subpopulation from different samples (Fig. 2).

\section{DATA SCALING}

Univariate histograms are commonly used for the simplest display of flow cytometry data. Histograms display the distributions of the events for one parameter of light scatter or relative fluorescence. They are useful for comparing the total number of cells in multiple samples that have been stained with the same marker or antigen of interest. Expression of positive antigen or fluorescence intensity of a sample can be distinguished by the distribution and quantitatively determined as percentage of cells above a certain threshold, or alternatively as the mean fluorescence intensity (MFI) of the antigen (see below). Most histogram analysis software also provides tools for overlaying histograms from several data files, allowing a rapid comparison of the parameter of interest. In bivariate plots, two parameters have to be discerned at the same time. Normally, dot plots are the most popular displays for this purpose, where each dot represents one event or cell. However, displaying bivariate data can influence interpretation, e.g., contour maps are relative insensitive to cell number which could mislead impression as some cells in particularly rare cells are excluded in the plots. For monochromatic (black and white) dot plots, there are several drawbacks of using these dot displays as different cell types expressing the same amount of fluorescence signals might be overlayed in the plot making it impossible to distinguish the two cell types. This is also particularly true when rare cell populations have to be analysed among a huge number of acquired cells. To overcome this so called outlier problem, using density or preferably coloured dot displays which give the graph a three-dimensional feel, may as well be recommended ${ }^{10,11,17}$.

In general, linear scaling is applied for fluorescence signals that vary 2 - to 10 -fold such as DNA distributions and cell cycle in which the amount staining fluorescence signal is proportional to the amount of DNA, it is therefore more logical to use linear scales for comparison. Linear scale is displayed as channel numbers, i.e., 0-1023, but if it is logarithmic (a 4 decade logarithmic amplification, for ex- 

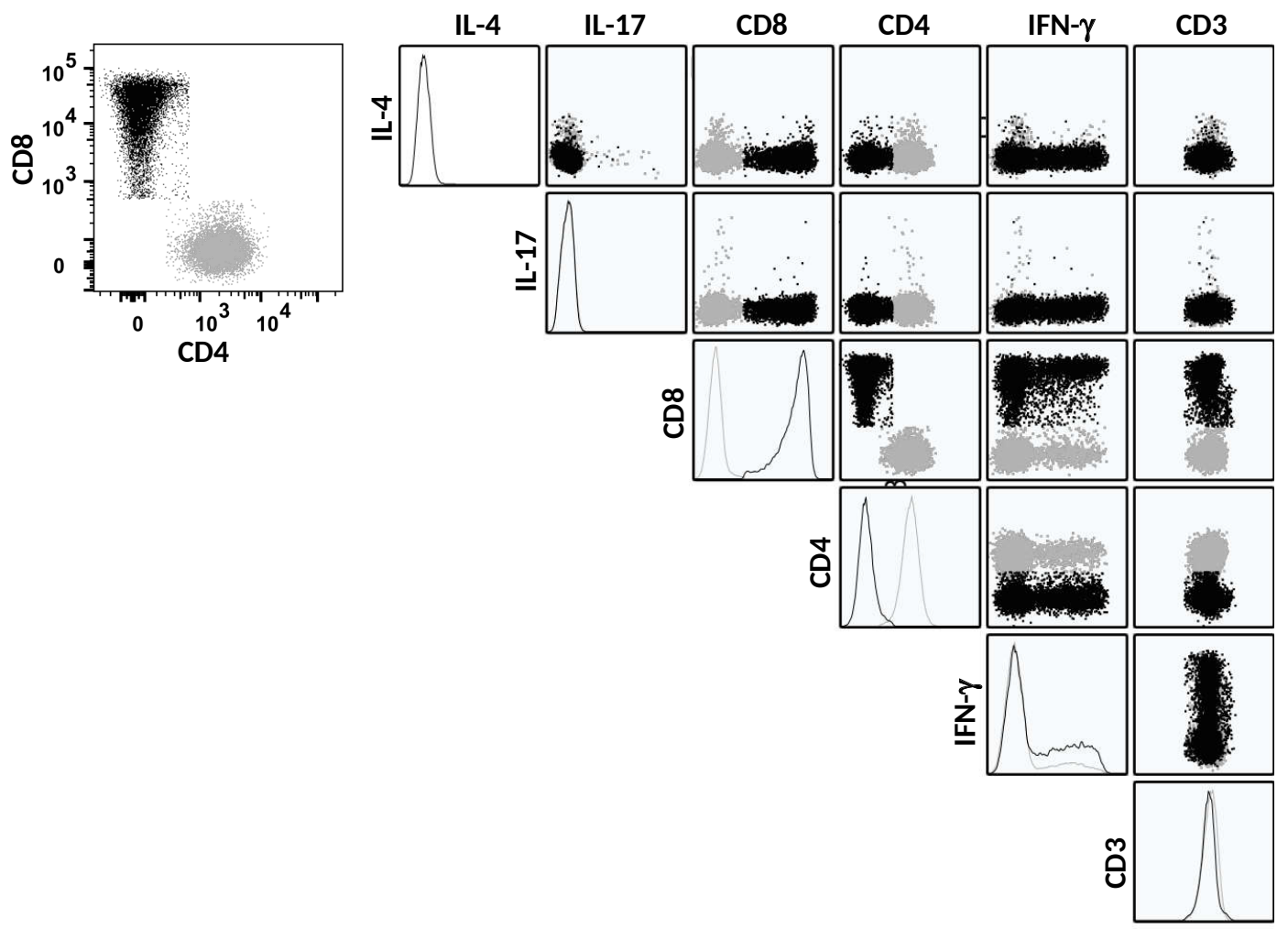

Fig. 2 Flow cytometric $\mathrm{N} \times \mathrm{N}$ plots display all parameters of selected subpopulations. The lymphocytes were stained with antibodies reactive to IL-4, IL-17, CD8, CD4, IFN- $\gamma$, and CD3. The IL-4, IL-17, and IFN- $\gamma$ expressions on CD4 T cells (grey) and CD8 T cells (black) are comparable by using multigraph overlays.

ample), it is as linear values of $1-10000$. Hence the $\log$ decade $10^{0}-10^{1}$ is equivalent to $0-255$ channel number or 1-10 linear values. To convert channel number to linear value, one can use the formula: $\log$ (linear value) $\times 256$ or vice versa, a channel number can be converted to linear value by using the formula: $10^{\text {(channel number) } / 256}$, e.g., if channel number is 255 , then the linear value is $10^{255 / 256} \sim$ 10 , or if the channel number is 1023 , the linear value will be $10^{1023 / 256} \sim 10000$. For data that have been acquired by linear amplification, channel numbers and linear values are equivalent, but for logarithmic amplified data, either channel numbers or linear values can be used as they are equivalent. Generally, the logarithmic scales used in the flow cytometer are 4 decade logs so the range is from $10^{0}$ to $10^{4}$ and each log decade takes up a quarter of the available channels. Apart from DNA analysis, linear scaling is also used for FSC/SSC light signals of white blood cell populations in immunophenotyping assay. Whereas the logarithmic scaling is valuable for immunofluorescence with wide dynamic ranges found on cell surface antigens and have fluorescence signals that are greater than 100-fold, e.g., fluorescence-labelled anti-CD3 antibody. Small cell populations such as red blood cells, platelets, and microparticles also require logarithmic scaling for their FSC/SSC light signals. In two-parameter displays, both logarithmic $x$ and $y$ axes have a fourto five-decade range, representing 10000 -fold from lower end to 100000 -fold of upper end of the scale. The logarithmic scaling compresses the channels of visual space as the scale increases, this often leads to visual misrepresentations of cell populations with negative fluorescence or minimal fluorescence that pile up on both axes especially in the zero channel. This could either due to fluorescence compensation error and/or fluorescence baseline subtraction error (Fig. 3a). To avoid misinterpretation of the data, one can either draw the gates or regions (see below) to cover cells that have been stacked up or use the newly adopted logicle or bi-exponential scaling approach ${ }^{11}$. This new scaling method is specially designed not only incorporating the useful feature of logarithmic displays but also transforming cell populations of low or background fluorescence with more accurate visualization (Fig. 3b). 

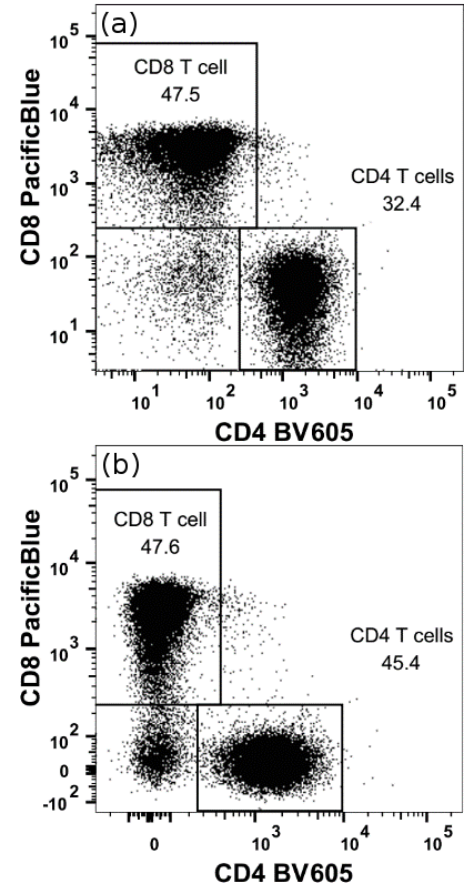

Fig. 3 Flow cytometric two-parameter dot-plots using logarithmic scaling display (a) and after applying logicle or bi-exponential display (b).

\section{DATA GATING}

The purpose of flow cytometry data gating is to draw gates or regions on light scatter plots of cell populations of interest for further characterization. A region is the normal term used for defining a cluster of cells preferably in two-parameter plot, whereas a gate is used for gating mixed populations. The first step in gating is typically to distinguish the cells based on their light scatter properties. Information of size and granularity, as well as fluorescence characteristics of cells has to be known prior to any gating. For instance, platelets and red blood cells are relatively small when compared to white blood cells, subcellular debris can be discriminated from single cells by relative size, estimated by FSC. Also, dead cells tend to have lower FSC and higher SSC than living cells. Lysed-and-washed whole blood cell analysis is the most common form of gating, and Fig. 4 depicts typical data gating of FSC versus SSC of whole blood using linear scaling analysis. The different light scatter signals of lymphocytes $\left(\mathrm{FSC}^{+} / \mathrm{SSC}^{+}\right)$, monocytes $\left(\mathrm{FSC}^{++} / \mathrm{SSC}^{++}\right)$, and granulocytes $\left(\mathrm{FSC}^{+++} / \mathrm{SSC}^{+++}\right.$) allow them to be easily distinguished from each other and from cell debris. Data can be analysed as histograms
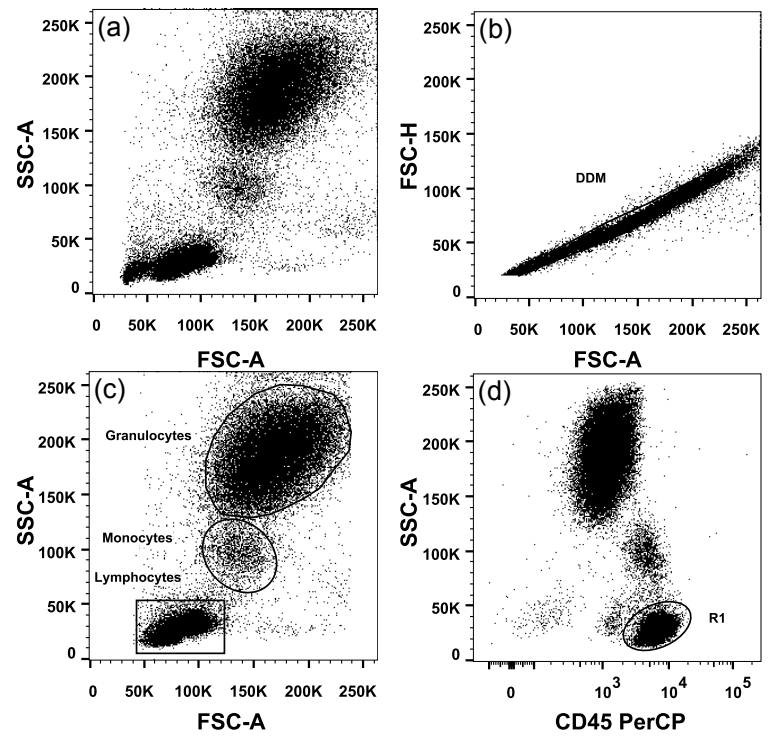

Fig. 4 Flow cytometric dot-plot data from human peripheral blood leucocytes stained with antibody reactive to CD45. (a) FSC/SSC light scatter of lymphocytes $\mathrm{FSC}^{+} / \mathrm{SSC}^{+}$, monocytes $\left(\mathrm{FSC}^{++} / \mathrm{SSC}^{++}\right.$) and granulocytes $\left(\mathrm{FSC}^{+++} / \mathrm{SSC}^{+++}\right)$. (b) Cell clumps or doublets outside each leucocyte populations can be eliminated by using Doublet Discrimination Module (DDM) of FSC-A versus FSC-H. (c) DDM provides improved representation of leucocyte populations of rectangular gated lymphocytes, ellipse gated monocytes and polygon gated granulocytes. (d) A region $\mathrm{R} 1$ has been drawn around the $\mathrm{CD} 45^{+++}$ lymphocytes.

or in two-parameter displays. On a histogram or univariate plot, a region is drawn to cover the whole histogram of interest. However, establishing regions on histograms can be somewhat subjective, if the histograms are heterogeneous. Proper controls, i.e., isotype control, biological control, are therefore essential for accurate gating. On a density plot, several styles of gating options are used; these include quadrant, rectangular, ellipse, polygon, and spider. There is no obligation to use many styles of regions in one plot. The customary approach to analyse the data is the use of marker method in which four quadrants, as an example, are created from which double negative cells, two single-positive cell populations, and double-positive cell populations for each marker can be readily determined. It should be noted that the coloured intensity displays are the most common way to represent a density plot. Each of the colour levels indicates increasing numbers of cells resulting from discrete populations of cells. However, it is a matter of preference, as 
sometimes, discrete populations of cells are also easily visualized on contour maps. Although there is no single best way to display data as each display style has its advantages and disadvantages, but be consistent with the style across all displays within an analysis.

Over the years, the FSC/SSC or morphological gating has been customarily used as the first plot for immunophenotyping assay by drawing a region around the desired cell cluster, e.g., lymphocytes and then gate the fluorescence expression on them. However, such FSC/SSC gating approach tends to be unreliable as all the desired lymphocytes may not be included in the region and undesired nonlymphocytes (monocytes, basophils and immature red blood cells) may be included. A more reliable approach for gating cells with their SSC (in linear scale in case of lymphocytes, and in logarithmic scale in case of red blood cells, as a few examples) versus their fluorescent antibody has been used to define the desired cells and used as a region. The advantage of using this gating approach is that only one region for each phenotype or marker, i.e., CD45 for all leucocytes with $\mathrm{SSC}^{+} / \mathrm{CD} 45^{+++}$for lymphocytes (Fig. 4d), or combined markers, i.e., $\mathrm{CD}^{+} / \mathrm{CD}^{+}$for T-helper cells, is required to define cells of interest. However, when there are several regions, data analysis can become complicated; a Boolean combination of several regions can be used to define a cell gate and its characteristics. To give an example of two cytokines' expression on $\mathrm{CD}^{+}{ }^{+}$T-cells that have been stimulated with stimulants. The two cytokines: IFN- $\gamma$ and IL-17 can be broken down to 8 populations that represent all combination of IFN- $\gamma$ and IL-17 using either 'And', 'Not' or 'Or' type of gate: e.g., IFN- $\gamma^{+}$And IL- $17^{+}$, IFN- $\gamma^{+}$Not IL- $17^{+}$, IFN- $\gamma^{+}$Or IL- $17^{+}$on stimulated $\mathrm{CD} 4^{+}$T-cells. A Boolean gating strategy such as that from FLOWJo software can be used to automatically generate these combinations. Details of certain cytokine-producing cells from these 8 IFN- $\gamma$ and IL17 combinations can be displayed as part of the gating hierarchy (see below).

One of the important grating strategies is to discriminate cell aggregation from single cell population. It is known that cells tend to aggregate to each other and become cell clumps. These cell clumps when passing through the laser take longer time than single cell or singlet; this in turn will affect the area of the light scatter signal. To deplete the cell clumps or doublets, a pulse geometry gate or Doublet Discrimination Module (DDM), e.g., FSCA versus FSC-H is applied to eliminate the doublets
(Fig. 4b,c). Another example of using this DDM is in the DNA analysis. A common feature of DNA analysis is the finding of cellular or event aggregates of doublets in which a doublet is formed when two cells or two nuclei with a $G_{1}$-phase DNA are mistakenly documented by flow cytometer as one event with a cellular DNA content similar to $\mathrm{G}_{2} / \mathrm{M}$ phase resulting in an overestimation of the number of cells in the $\mathrm{G}_{2} / \mathrm{M}$ phase of the cell cycle. Another good feature in flow cytometric gating strategy is the 'back gating' tool, a tool that allows the data inspection to determine what cells would fall in the final population, assuming the gate of interest was not used in the gating scheme. This can be achieved by applying a given gate to see where the population is with respect to the total population. It also allows for confirmation that a given gate or region is appropriately drawn. This is so important, especially for analysing rare cell population samples where there are a lot of cells that would be included in the final gate and are positive for a viability marker.

\section{DATA INTERPRETATION}

Flow cytometric data are numerous particularly data that are generated by polychromatic flow cytometer, thus finding the best way to compare those data can be challenging. In general, a positive population of cells with any immunofluorescent markers from any flow experiments can be presented as population measurements, e.g., percent of $\mathrm{CD} 4^{+}$T-cells or it can be expressed as MFI, e.g., MFI of IL-17 expression on activated CD4 ${ }^{+}$T-cells. Nowadays, most flow cytometers provide software that automatically generate the percent positive cell population, i.e., the BD MULTISET and FACSDIVA software. For MFI, this is the measurement of the level of florescence distribution of a cell population, e.g., when comparing the level of fluorescence from two fluorescent distributions. To quantify MFI data, one can measure the central fluorescent tendency by using the modal, mean, or median channel. In the ideal situation, the fluorescent data are normal distributions, thus the mean, median and mode are very similar. But in reality, the fluorescent data are not normally distributed, the more that the data skew, the further the mean MFI drifts towards the direction of fluorescent skew. In the linear scaling data, both the mean and median of MFI are used to quantitate cellular MFI, i.e., a cell population with a linear value of 200 is 20 times brighter than a cell population with a linear value of 10 . But in the logarithmic amplified data, arithmetic 

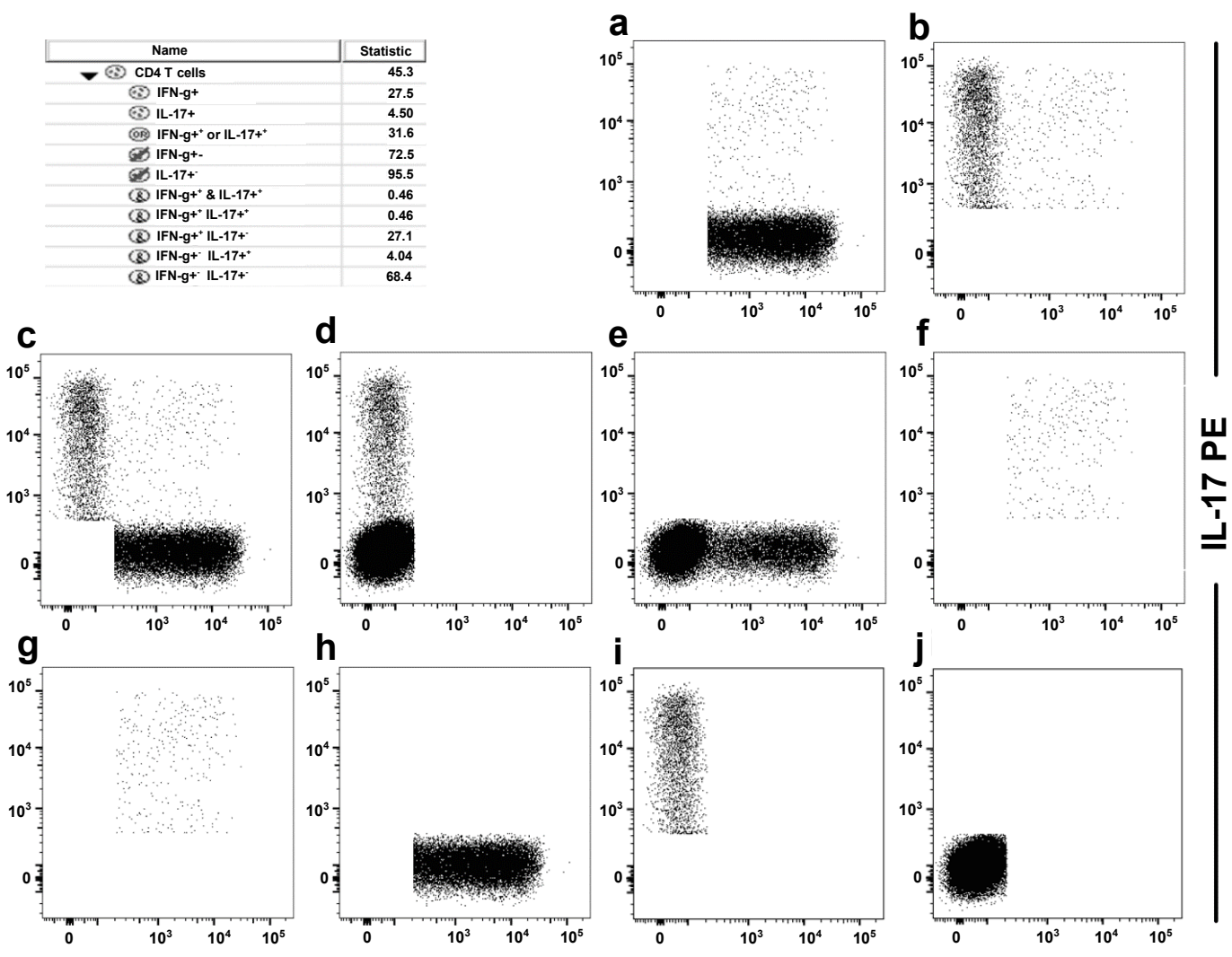

IFN-g APC

Fig. 5 A series of Boolean gates that represent all combinations (plus and minus) of IFN- $\gamma$ and IL-17 expressed on stimulated $\mathrm{CD}^{+}$T-cells. A table shows statistics of combination gates applied to IFN- $\gamma$ and IL-17 on CD4 T-cells.

mean becomes less representative of the data, it is the geometric mean that is often used as it takes into account the weighting of the data distribution. However, if the geometric mean shows significant shifts, then the median is recommended as it is less influenced by skew distribution. It should be noted that if the fluorescent data are a bimodal distribution, a continuous probability distribution with two different populations or modes, then gating each population and presenting as the percentage value is easier as well as more statistically significant.

As mentioned above, polychromatic flow cytometry provides us with the ability to defy many discrete subsets of the immune system. For example, determination of the frequency distribution of naïve, central memory, effector memory and effector cells of $\mathrm{CD}^{+}$and $\mathrm{CD} 8^{+}$T-cells in peripheral blood mononuclear cells (PBMC) stimulated with viral antigen plus cytokine, i.e., IL15. Stimulated and unstimulated PBMC are stained using 8-colour immunostaining panel consisting of human T-cell phenotype panel consisting of CD3, CD8, CD27, CD28, CD45RA, CD57, CCR7 and a dump channel cocktail of monocyte (CD14) and Bcell (CD19) antibodies plus one live/dead marker of 7-aminoactinomycin-D (7-AAD) or a panel using CD3, CD4, CD8, CD45RA, CD45RO, CCR7, CD62L with a dump channel antibodies and 7AAD. Viable $\mathrm{CD} 14^{-} / \mathrm{CD} 19^{-} / 7-\mathrm{AAD}^{-}$are gated through $\mathrm{CD}^{+} \mathrm{T}$-cells. These pan T-cells are further dissected into $\mathrm{CD}^{+}$and $\mathrm{CD}^{+}$T-cells. The events in the $\mathrm{CD}^{+}$and $\mathrm{CD} 8^{+}$regions are then interrogated by the remaining 4 surface markers to determine as many as 16 possible discrete subpopulations of $\mathrm{CD}^{+}$and $\mathrm{CD} 8^{+} \mathrm{T}$-cells, e.g., naïve $\left(\mathrm{CD} 45 \mathrm{RA}^{+} \mathrm{CD} 45 \mathrm{RO}^{-} \mathrm{CCR}^{+} \mathrm{CD}^{-} \mathrm{L}^{+}\right)$, central memory $\left(\mathrm{CD} 45 \mathrm{RA}^{-} \mathrm{CD} 45 \mathrm{RO}^{+} \mathrm{CCR} 7^{+} \mathrm{CD}^{+} 2 \mathrm{~L}^{+}\right)$, effector memory (CD45RA ${ }^{-} \mathrm{CD} 45 \mathrm{RO}^{+} \mathrm{CCR}^{-} \mathrm{CD}^{-} 2 \mathrm{~L}^{-}$), and effector $\left(\mathrm{CD}^{4} 5 \mathrm{RA}^{+} \mathrm{CD}^{4} 5 \mathrm{RO}^{-} \mathrm{CCR}^{-} \mathrm{CD}^{-} \mathrm{CL}^{-}\right)$). This so called, hierarchical gating provides the best gating strategy to identify target populations and to determine the subphenotype frequencies from each 


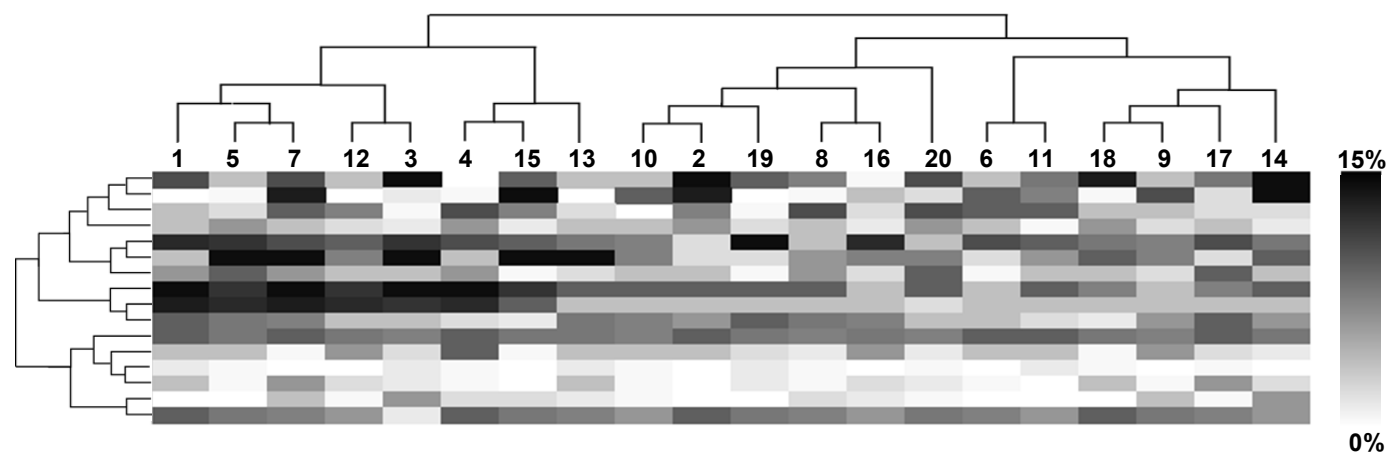

Fig. 6 Multivariate dataset analyses of 16 subphenotypes of each of the 20 samples defined by FCOM (Winlist) data reduction were analysed by average-linkage hierarchical cluster analysis. Differences in frequency distribution among subphenotypes expressed on the samples were indicated by colour shading.

sequential dot-plots. However, this procedure is very complicated and time-consuming if multiple testing samples are determined as might occur in clinical settings involving many patients in different treatment protocols. To simultaneously show pattern distributions and compare statistics on these T-cell subpopulations under many variables: e.g., treatment protocols, age group, etc. Simple and easy graphical displays in the forms of bar charts or pie charts are therefore required. At present, a freely-available US Government supported application named SPICE (Simplified Presentation of Incredibly Complex Evaluations) which works on Apple Mac-based software is used along with FLOWJo background subtraction and formatting of exported data software. SPICE is a user-friendly data mining software tool that analyses and organizes large data files from polychromatic flow cytometry and displays the normalized data graphically. The software enables users to study potential correlations in their experimental data within complex data sets ${ }^{18}$. In certain circumstances, there are some subpopulations in the analysis of many multivariate datasets that are less important as they exhibit very low frequencies and weak positive staining ${ }^{11,19}$, such as T-cells that are not naïve, central memory, effector memory and effector cells, but they are Tcells with and CD45RA ${ }^{+} \mathrm{CD}^{2} 5 \mathrm{RO}^{-} \mathrm{CCR}^{-} \mathrm{CD}^{-} \mathrm{L}^{+}$

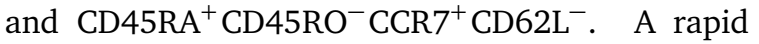
and effective approach for reduction and analysis of these superfluous data in the complex multivariate analysis is therefore critical. There are several available analytical tools using automated data elimination analysis on hierarchical gating to interpret and compare the frequency patterns of distribution. Among them hierarchical clustering analysis using FCOM, a 'combination function' data reduction and cluster analysis software from Winlist (Verity House Software) is the most common method as it allows for an easy visual representation of the data ${ }^{20}$. This hierarchical clustering analysis algorithms are similar to the software tools used in a wide diversity of gene expression studies ${ }^{21-23}$. Cells analysed by polychromatic flow cytometry are divided into multiple subpopulations, the number of cells within each subphenotype is provided as a relative frequency of the total cell population of interest (Fig. 6). This method is very useful and more organized approach to cluster analysis of large subpopulation arrays such as 1024 datasets in a 10colour panel.

In conclusion, a number of practical considerations have been described when analysing the complex datasets generated from the polychromatic flow cytometry. These guidelines are also applied to simple two to four-colour experiments.

Acknowledgements: This study project is supported by the Thailand Research Fund-Distinguished Research Professor Grant (DPG5980001). LK is supported by Chalermprakiat Foundation, Faculty of Medicine Siriraj Hospital, Mahidol University.

\section{REFERENCES}

1. Herzenberg LA, Sweet RG, Herzenberg LA (1976) Fluorescence-activated cell sorter. Sci Am 234, 108-17.

2. Van Dilla MA, Dean PN, Laerum OD, Melamed MR (1985) Flow Cytometry, Instrumentation and Data Analysis, Academic Press, Orlando, FL.

3. Wood JCS (1993) Clinical flow cytometry instrumentation. In: Bauer KD, Duque RE, Shankey TV (eds) Clinical Flow Cytometry. Principles and Applications, Williams and Wilkins, Baltimore, MD, pp 71-92. 
4. Shapiro HM (2003) Practical Flow Cytometry, 4th edn, Wuley-Liss, Hoboken, NJ.

5. Chattopadhyay PK, Roederer M (2010) Good cell, bad cell: flow cytometry reveals T-cell subsets important in HIV disease. Cytometry A 77A, 614-22.

6. Craig FE, Foon KA (2008) Flow cytometric immunophenotyping for hematologic neoplasms. Blood 111, 3941-67.

7. Roederer M, Kantor AB, Parks DR, Herzenberg LA (1996) Cy7PE and Cy7APC Bright new probes for immunofluorescence. Cytometry 24, 191-7.

8. Chattopadhyay PK, Price DA, Harper TF, Betts MR, Yu J, Gostick E, Perfetto SP, Goepfert P, et al (2006) Quantum dot semiconductor nanocrystals for immunophenotyping by polychromatic flow cytometry. Nat Med 12, 972-7.

9. Roederer M, Moody MA (2008) Polychromatic plots: graphical display of multidimensional data. Cytometry A 73A, 868-74.

10. Lugli E, Roederer M, Cossarizza A (2010) Data analysis in flow cytometry: the future just started. Cytometry A 77A, 705-13.

11. Herzenberg LA, Tung J, Moore WA, Herzenberg LA, Parks DR (2006) Interpreting flow cytometry data: a guide for the perplexed. Nat Immunol 7, 681-5.

12. Pattanapanyasat K, Yongvanitchit K, Tongtawe $P$, Tachavanich K, Wanachiwanawin W, Fucharoen S, Walsh DS (1999) Impairment of Plasmodium falciparum growth in thalassemic red blood cells: further evidence by using biotin labeling and flow cytometry. Blood 93, 3116-9.

13. Pattanapanyasat K, Phuang-Ngern Y, Sukapirom K, Lerdwana S, Thepthai C, Tassaneetrithep B (2008) Comparison of 5 flow cytometric immunophenotyping systems for absolute CD4+ T-lymphocyte counts in HIV-1-infected patients living in resource-limited setting. J Acquir Immune Defic Syndr 49, 339-47.

14. Khowawisetsut L, Pattanapanyasat K, Onlamoon N, Mayne AE, Little DM, Villinger F, Ansari AA (2013) Relationship between IL-17 synthesizing subsets, Tregs and plasmacytoid dendritic cells that distinguish among elite controllers, low, medium and high viral load rhesus macaques following SIV infection. PLoS One 8, e61264.

15. Angkasekwinai P, Sringkarin N, Supasorn O, Fungkrajai M, Wang YH, Chayakulkeeree M, Ngamskulrungroj P, Angkasekwinai N, et al (2014) Cryptococcus gattii infection dampens Th1 and Th17 responses by attenuating dendritic cell function and pulmonary chemokine expression in the immunocompetent hosts. Infect Immun 82, 3880-90.

16. Spidlen J, Moore W, Parks D, Goldberg M, Bray C, Bierre P, Gorombey P, Hyun B, et al (2010) Data file standard for flow cytometry version FCS 3.1. Cytometry A 77A, 97-100.

17. Lugli E, Troiano L, Cossarizza A (2009) Investigating $\mathrm{T}$ cells by polychromatic flow cytometry. Meth $\mathrm{Mol}$
Biol 514, 47-63.

18. Roederer M, Nozzi JL, Nason MC (2011) SPICE: exploration and analysis of post-cytometric complex multivariate datasets. Cytometry A 79A, 167-74.

19. Tung JW, Parks DR, Moore WA, Herzenberg LA, Herzenberg LA (2004) New approaches in fluorescence compensation and visualization of FACS data. Clin Immunol 110, 277-83.

20. Petrausch U, Haley D, Miller W, Floyd K, Urba WJ, Walker E (2007) Polychromatic flow cytometry: a rapid method for the reduction and analysis of complex multiparameter data. Cytometry A 69A, 1162-73.

21. Kohlmann A, Schoch C, Schnittger S, Dugas M, Hiddemann W, Kern W, Haferlach T (2003) Molecular characterization of acute leukemias by use of microarray technology: genes chromosomes. Cancer 37, 396-405.

22. Istepanian RSH, Sungoor A, Nebel J-C (2011) Comparative analysis of genomic signal processing for microarray data clustering. IEEE Trans Nanobiosci 10, 225-38.

23. Guerra-Laso JM, Raposo-García S, García-García S, Diez-Tascón C, Rivero-Lezcano OM (2015) Microarray analysis of Mycobacterium tuberculosis-infected monocytes reveals IL26 as a new candidate gene for tuberculosis susceptibility. Immunology 144, 291-301. 